\title{
Capturing Digital Experience: The Method of Screencast Videography
}

\section{Dr Fatema Kawaf}

Senior Lecturer in Digital Marketing

Greenwich Business School

University of Greenwich

Maritime Greenwich Campus

Old Royal Naval College

London SE10 9LS

$\mathrm{T}+44(0) 2083319060$

E-mail: F.Kawaf@greenwich.ac.uk 


\begin{abstract}
This paper presents a novel research methodology, screencast videography (SCV), as an approach to studying interactions and experiences in the digital space. Screencasting is a method of digitally-recorded computer/mobile screen output, with or without audio narration. Focusing on the dynamic, highly visual digital environment in which many modern experiences such as e-shopping take place, SCV can be used for videographic studies of digital experiences that are rarely captured by means of traditional videography owing to the private settings of such experiences. $\mathrm{SCV}$ is able to capture dynamic experiences in the digital space, opening up opportunities for a wealth of screencast-based research to enhance our understanding of digitallyoccurring interactions, experiences and phenomena. This paper discusses the ontological and epistemological assumptions of SCV and how it is situated in relation to other relevant methodological approaches (videography and netnography). It then outlines, step-by-step, the methodological protocol for SCV and its possible applications. An illustrative example of using this method to study digital experience in the context of online fashion shopping is presented and discussed. This is the first presentation of such a method, offering a promising approach to studying similar experiences in the digital world.
\end{abstract}

Keywords: videography, screencast, digital experience, visual research 


\section{Introduction}

In today's fast-paced, data-rich, dynamic, social and visual world, many of our experiences, activities and daily interactions have shifted slowly but surely to the digital realm. Naturally, the research interest in studying the digital experiences of shopping, consumption, socializing, dating, gambling, and searching for information, ideas and solutions has also increased (e.g. Bilgihan, 2016; Chaney, Lunardo \& Mencarelli, 2018; Kawaf \& Tagg, 2012; Pantano \& Priporas, 2016; McLean \& Wilson, 2016).

A plethora of methodological solutions have been offered in response to increasing demand for big data and ever-growing online content (Kannan \& Li, 2017). Yet, methodological solutions to in-depth qualitative understandings of phenomena, such as digital experiences and interactions, have been limited to various adaptations that often dismiss the dynamic, visual nature of the digital environment (Kawaf \& Tagg, 2017). Notably, the widespread success of netnography (Kozinets, 2015) has illustrated the need for such a method. However, as this paper argues, various qualities of the digital experience - consumer journeys, dynamic interactions, and navigations of the environment, to name just a few - have rarely been captured using existing methods. The equivalent of a fully-immersive, highly-visual, video-ethnography is still not available for the study of experiences in the digital realm.

Accordingly, this paper presents screencast videography as a novel methodological approach to analysing digital experiences and interactions. Screencast videography is defined here as $a$ research method that adopts a dynamic visual form of inquiry. It is philosophically underpinned by the ontology of the moving image. The method uses screencasts - videos of screen activities or outputs - as its main mode of data collection. The screencast videos capture dynamic onscreen interactions and experiences as they occur. This helps offer detailed records of online 
experiences (e.g. online shopping, information search, dating, video gaming, gambling, etc.) that are not usually observable using conventional methods. Screencast videography focuses on capturing computer-mediated or digitally-occurring experiences in their dynamic 'moving' form, rather than static or still instants from among such experiences.

Screencasting, to use the technical term, refers to the "method of presenting digitally recorded playback of computer screen output which often contains audio narration" (Brown, Luterbach, \& Sugar, 2009: 1748). Using screen capture or screen recording 'casting' software, it is possible to record the screen of any electronic device, such as a smartphone or a laptop. The resulting screencast typically shows a video of the screen, recorded with any content displayed on it, as well as any activity which has taken place, such as navigation, typing, etc.

Screencasting is a popular tool for producing yideos of on-screen activities. As an example, Mendeley (2014) offered this $\underline{v i d e o}^{1}$ of a screencast explaining how to install and use software for citation and reference management. Screencasts have become extremely popular in virtual learning environments due to their simplicity and effectiveness in explaining 'how to' situations, such as explaining how to use Mendeley in the video above (Brown et al., 2009).

In addition, away from explaining on-screen procedures and use in 'how to' contexts, screencasts have also proved popular among various video gaming communities. Numerous videos can be found on YouTube and Vimeo of video gamers recording and sharing their games and techniques in passing levels, etc. This $\underline{\text { screencast }^{2}}$ is of a Grand Theft Auto (GTA 5) videogame player, who is showing off his purchase of a luxury yacht within the game for

\footnotetext{
${ }^{1}$ Mendeley Referencing Screencast Video https://www.youtube.com/watch?v=Gv6 HuCYExM

${ }^{2}$ GTA Gaming Screencast Video https://www.youtube.com/watch?V=VFnQUqEr5VY
} 
' $\$ 28,000,000$ '. The shopping spree appears to have been a very exciting experience, with followers immediately fantasising in the following ways:

Commenter 1: “Why isn't this real life? 2 wow wouldn't life be cool if you actually owned your own boat?"

Commenter 2 (replying): "Life sucks. That's why we play games".

The examples above do not show screencasting as a research method. In its current format, screencasting is mainly used by educators and trainers in a 'how to' context, as a tool for explaining screen-related activities, and by amateurs showcasing their video gaming techniques and purchases. However, screencasting also has great potential for use in research contexts, particularly in studying digital experiences. As such, screencast videography as a research method uses screencasting as the main method of data collection in studying digital experiences by capturing the on-screen interactions in lived-through, computer-mediated or digitallyoccurring experiences.

The next section of this paper discusses the ontological and epistemological aspects of SCV, clearly situating the method in relation to the closely-linked methodological approaches of netnography and videography. Section 3 provides a step-by-step guide to the methodological protocol, and discusses the theoretical foundations, applications and implications of this method. Section 4 presents an illustrative example of the method in use, and Section 5 explores the theoretical and managerial implications of SCV as a research method, and its possible future directions. 


\section{Positioning screencast videography}

Screencast videography's focal point is the study of digital experiences. Therefore, this section reviews the state-of-the-art of the literature associated with experience in both offline and online contexts. Then, the section moves to position screencast videography in relation to other existing methodological approaches.

The rise of 'experience' in consumer research is most notable in the early work of Holbrook and Hirschman (1982), in which they presented an experiential view focusing on the symbolic, hedonic and aesthetic nature of consumption, as opposed to the information processing model of the rational consumer (Bettman, 1980). Indeed, Hirschman and Holbrook's (1982) work remains the central reference point for experience research in Marketing; the roots of this concept are traceable back to the work of early philosophers. Indeed, Holbrook (2006: 715) has since acknowledged that Holbrook and Hirschman (1982) did not 'invent' the concept of experience, which goes back to the early works of "Sid Levy at Northwestern in the 1960s, Wroe Alderson at Wharton in the 1950s, and the economists Lawrence Abbott in the 1950s or Alfred Marshall in the early 1900 s...".

In fact, an early twentieth century philosopher, William James, defined experience from the anti-dualistic and anti-Cartesian philosophy of pragmatism as "a continuous stream of consciousness" (1907). James suggested that, like a stream of water that is always moving forward, experience is never static and never still. In consumer research, Thompson, Locander, and Pollio (1989) took a similar stance by presenting existential phenomenology as a paradigm for studying consumer experience, thus offering a different perspective from the Cartesian view which was dominant at the time. 
Such early roots of the concept of experience enrich its multidisciplinary nature, but it arguably does not always serve a positive purpose in experience research, as it deters unity on the very essence of what an experience is (Petermans, et al., 2013). Indeed, as Carù and Cova (2003) asserted in the context of marketing and consumer research, definitions of experience are not unitary, as each perspective has relied on one of the varying roots of experience, from philosophy to psychology to sociology and anthropology. Inevitably, this multidisciplinary nature of the term results in varying methodological approaches being taken. In addition to quantitative methods, with the rise of Consumer Culture Theory (Arnould and Thompson, 2005), various ethnographic and netnographic approaches to experience research have appeared to employ participant observation, in-depth interviews, narratives, diaries, images and video methods to communicate a compelling, resonant story about the consumption experience (Chaney et al., 2018).

Indeed, experience literature in consumer research has flourished, inspired by the design of the environment; for example, Schmitt (1999) designed his experience framework of the five senses, and Pine and Gilmore (1998) introduced the concept of the four realms of experience. Furthermore, a wealth of literature on experience in marketing and consumer research has focused on the social and cultural values and properties of experience. For instance, Arnould, Price, and Tierney (1998) moved beyond the relationships between expectations and satisfaction to uncover seyeral pillars of an extraordinary experience: interrelationships between the narratives of harmony with nature, connections with others, and personal renewal. On the other hand, Tumbat and Belk (2011) discussed the very individualistic and competitive nature of extraordinary experiences, as opposed to feelings of community, while Scott, Cayla, and Cova (2017) presented painful extraordinary experiences as a form of escapism. 
In addition, a wide range of research has focused on retail and shopping experiences, thus contributing to our understanding of the psychological, sociological and cultural dimensions of experience. Examples of this strand of research include the experience of flagship stores such as ESPN Zone Chicago (Kozinets, Sherry, DeBerry-Spence, Duhachek, Nuttavuthisit, \& Storm, 2002), American Girl Place and its linking to both materialism and heroism (Borghini, Diamond, Kozinets, McGrath, Muñiz, \& Sherry, 2009), and the spectacular experience of Nike Town (Peñaloza, 1998).

Such research has studied consumption experiences from cultural and societal perspectives and engaged in a variety of rich methodological approaches to ethnography using methods including participant observation, in-depth interviews, life narratives, diaries, images and videos (Chaney et al., 2018). The accumulated knowledge on the various types of experiences studied in offline contexts has enriched understanding of various types of 'offline' experiences and their interrelationships with social and cultural values. However, in crossing into the digital realm, experience research to date has tended to focus merely on design aspects (e.g. Bilgihan, 2016; McLean \& Wilson, 2016; Rose et al., 2011; 2012), often overlooking the individualistic nature of experience (Kawaf \& Tagg, 2017) as a unique but shapeable story constructed by the individual (Hassenzahl, 2010). This is because the central focus of this type of research has often been the close examination of the environment and customers' responses to it. Customers are ultimately viewed merely as organisms responding to external stimuli; thus, such research has rarely fully engaged with the nature of the experience itself.

Hassenzahl (2010) highlighted that, despite its great contribution, design research and its focus on experience from an environmental perspective has limited our understanding of experience as a concept. He offered the following insight (ibid.: 1): 
Experience is a chunk of time that one went through - with sights and sounds, feelings and thoughts, motives and actions; they are closely knitted together, stored in memory, labelled, relived and communicated to others. An experience is a story, emerging from the dialogue of a person with her or his world through action.

This perspective stemming from James's (1907) notion of experience as a stream of consciousness also highlights the dynamic nature of experience. However, as Rylander (2012: 11) explains:

We tend to overlook the continuity of the stream of thought because we typically focus on the substantive parts rather than the transitive parts. Although we cannot express the qualities of the latter in words, it does not mean they are not important.

The evident inability to capture or examine the dynamics and continuity of experiences is perhaps a shortcoming of current methodological approaches. As James (1907) argued, the whole of an experience is bigger than the sum of all its parts put together, as its transitive and dynamic qualities are lost in the affordances of current methodological approaches. For instance, in the context of digital experience, the last decade has witnessed a burgeoning and widespread use of netnography as "a qualitative research methodology that adapts ethnographic research techniques to study the cultures and communities that are emerging through computer-mediated communications" (Kozinets, 2002: 62).

Netnography has been improved and adapted in order to better suit the digital realm of dynamic interactions, rapid decisions and unfolding events. Kozinets (2015) redefined the method, focusing on social media, communications, and the connections that people make online, asking questions about the nature of online social experiences and interactions, and 
delving deeper into cultures, communities, gatherings, and experiences in order to tell the whole story.

A wealth of literature has adopted and adapted netnography over the last two decades (Bartl, Kannan and Stockinger, 2016; Heinonen and Medberg, 2018, Lugosi and Quinton, 2018). For instance, García-Álvarez, López-Sintas and Samper-Martínez (2017) adopted a netnographic approach to studying Gamers Restaurant City to study gamers' experiences of being part not only of the game, but also its Facebook community, and the aftermath of the game closure. Moreover, other examples of netnographic studies which have focused on experience include the 'salsa dancing' experience (Hamilton \& Hewer, 2009) and the 'running and music' experience (Kerrigan, Larsen, Hanratty and Korta, 2014). All these examples have demonstrated how netnography enables understanding of such experiences based on the online interactions and conversations in relation to those topics. As such, the netnographic approach to studying experience remains limited to the conversations ayailable online rather than the journey of the experience itself.

A review of the netnography-based literature indicates that most netnographies tend to focus on text and the analysis of text-related interactions and conversations rather than visual ones (Lugosi \& Quniton, 2018). Arvidsson and Caliandro's (2016) netnography on brand public and Kozinets, Patterson and Ashman's (2016) netnography on food porn are two of the few visual based netnographies to have studied videos and images posted on multiple virtual platforms. As such, netnography research addressing the visual nature of the digital environment has been limited, and the research exploring the interactions with this digital, visual and dynamic environment is mostly inapplicable using netnography. 
Acknowledgment of the highly interactive and highly visual nature of the online environment is paramount in digital experience research, and netnography has shortcomings in relation to addressing navigation in, and interactions with, the digital environment. As Lugosi and Quinton (2018: 302) observed:

The mapping of actors, be they human and or non-human and the networks they create across devices, platforms and technologies requires acknowledgement of the fluid migration back and forth of people and their behaviour in relation to technology. The dynamic possibilities being adopted for interaction need to be recognised and accounted for rather than offering fixed accounts of online behaviour.

Whilst recent research has shown an interest in furthering netnography in order to enable better understanding of the interactions and the mapping of human and nonhuman actors, much work still needs to be done to address the visual nature of this environment as well as the dynamic interactions. Kedzior (2014: 86) explained that:

Online virtual worlds are complex environments: simulated and animated, mediated and interactive. Consequently, observational methods, which are the crux of ethnographic methodology, cannot be directly applicable. Researchers who intend to study these online phenomena have to prepare themselves accordingly prior to entrée into the field. They have to amass knowledge about the architecture of an online virtual world (i.e. the way in which it is organized) to be able to put into context its avatars' actions, behaviours, and customs.

Indeed, as Kedzior (2014) has argued, observational methods, which represent the roots of ethnographic methodology, have not been possible or directly applicable in the context of online experiences. The very possibility of not only observing but also video recording individuals as they immerse themselves in their digital environment was not possible prior to the introduction of screencast videography. 
Whilst netnography establishes a protocol for studying digital human interactions with people, brands, technologies, and so on (Kozinets, 2002; 2015), it focuses mainly on digital footprints. The netnographic researcher captures and analyses what the digital user chooses to leave behind in the digital realm, in the form of comments, posts, shared links, and any other types of interaction that can be observed after being made. For instance, in Kozinets's (2002) illustrative example of an online coffee newsgroup, it is obvious that what users post online can be analysed and captured, thus revealing important insights into interactions with brands and with other users. However, netnography does not capture all the interactions, processes, attempts to comment, comments deleted before posting, and other important dynamic interactions that do not result in a digital posting of some kind. Arguably, this gap leads to a loss of nuance and an incomplete telling of the story, as netnography can only study tangible actions online, e.g. a post or a comment. Kozinets, Scaraboto and Parmentier commented (2018, p.236) that:

A netnography is a kind of selfie. Being a netnographer means saving a slice of social reality as it is reflected in your life and your thoughts. Just as it is required with a selfie, the image of the image-taker must be a part of the overall picture. The netnography is an exploration of a time in your life, a set of communications and a social situation, that will never again exist exactly as it does when you capture them.

Despite the excellent insights enabled by netnography, a few questions remain unanswered. If, as the above quotation suggests, netnography's snapshot of communication at a slice of time "will never again exist exactly as it does when you capture them", then there is a chance that such communications are not identical to when they were produced either. This possibility may not be an issue when the research intends to study only what is left behind on the screen, such as comments and posts. However, experience research has often been interested in processes and 
customer journeys, rather than simply examining what is left behind from the experience.

Arguably, this means that netnography falls short of fully capturing such a journey and grasping the dynamic nature of experience. If netnography is 'a kind of a selfie', then the fact that taking a selfie is a journey in itself is problematic, as people often take numerous selfies from various angles, and under different lights and filters, in order to arrive at the 'perfect selfie', and share it. Netnography does not capture the processes that happen behind that single shared selfie, just as it does not capture what goes on behind the screen as an individual consumes and interacts with the digital environment. Instead, netnography captures what the individual chooses to leave as a digital footprint; capture-able actions, such as posts, comments, likes, follows, etc.

The limitation described above exemplifies the loss of meaning and the gap in knowledge associated with James's (1907) notion of experience as a stream and Rylander's (2012) argument that, methodologically, research appears to have focused on the substantive parts of an experience rather than on the transitive parts, thereby losing a dynamic understanding of digital experiences in this context. In the case of netnography, a fully dynamic understanding of experience cannot be attained simply by focusing on the digital footprint actively left in relation to it. Toraldo, Islam, and Mangia (2016) referred to this epistemological gap as the challenge of converting lived experience into elusive knowledge, focusing particularly on the tacit, aesthetic and embodied aspects of organisational life and experience. These authors also discussed the potential affordances of videography as a scaffold upon which to translate tacit, aesthetic and embodied experiences multimodally into elusive knowledge that is unattainable using other textual methods. Again, this ties in closely with the dynamic nature of experience, and with the current methodological approaches' potential for loss of meaning, and telling of incomplete stories. 
Video-based research has the ability to capture the live interactions and dynamics of an experience. Lemke (2007: 40) suggested that video is "a space of visible and audible dynamic activity", which has temporal affordances and produces permanent and accessible observations of an experience, thus addressing the need for a dynamic approach to studying experience, and producing records that allow full immersion in the data for the purposes of analysis (Belk, Caldwell, Devinney, Eckhardt, Henry, Kozinets, \& Plakoyiannaki, 2017). The multimodality of videography also provides a basis for translating the aforementioned tacit, aesthetic and embodied aspects of experience into elusive knowledge (Toraldo et al., 2016), by offering "a sense of understanding that is novel and interesting, evocative and emotionally engaging" (Belk et al., 2017: 3).

Videography's ontology of the moving image (Rokka, Hietanen, \& Brownlie, 2017), which is always moving forward and never still, ties in very closely with James's (1907) and Rylander's (2012) notions of experience as a constantly moving stream. Rokka and Hietanen (2018: 10) recently observed that:

Videographic research, by tapping into the affective and evocative capacities of the moving image, should foreground its phantasmatic qualities (Žižek, 2006) and actively embrace affective and 'sensory ways of knowing' (Toraldo et al., 2016: 2) and encounters it produces.

Videography affords access to the elusive knowledge embodied in everyday experiences (Toraldo et al., 2016), as it offers opportunities not only for detailed records of dynamic experiences, but is also able to "empathetically comprehend the embodied experiences of those represented" (Pink, 2007: 248). Pink (2011) further emphasised the ontology of the moving image by explaining how our experience of moving through the world is one of knowing 
(moving forward), and that knowing is created by moving. Products and participants in wider environments are both produced by, and consumed in, movement.

Despite videography's ability to capture the dynamic aspect of experience and its "resonant, emotional, vibrant and humanizing" qualities (Kozinets \& Belk, 2007: 340), the method is not well-suited to a digital context because digital experiences cannot be video-recorded. If videography were to be employed in an online shopping experience context, for instance, by placing a camera in front of online shoppers, the results would be likely to capture only their bodily postures and facial expressions. This approach would not capture the live dynamic experiences of these people, and insights into their actions, interactions and behaviours would be lost. Indeed, a wide gap exists between the depth and resonance achieved in videographic experience research, and the studies of digital experiences for which, to date, a videographic approach has been impossible. As Belk et al. (2017: 14) have suggested:

With increasingly networked online data, virtual and augmented reality, consumer research videography will move our knowledge portrayals to new places. Our research representations will become more immersive, more collective, more active, more dynamic and, perhaps, more overwhelming [...] Although it is impossible to predict exactly how consumer researchers may be able to take advantage of all the new opportunities to share narratives for investigation and discovery, the future of videography will be filled with new opportunities to do so.

In this view, screencast videography is presented as an evolutionary research method that encompasses the abilities of videography as a dynamic research method in capturing the dynamism and live interactions of computer-mediated and digitally-occurring on-screen experiences, and can translate the tacit, aesthetic and embodied aspects of experience into elusive knowledge. Screencast videography therefore moves beyond the 'static' approach of 
netnographic research and studies of digital footprints towards a dynamic methodology, thus facilitating the storytelling of dynamic digital experiences and live online interactions. It takes the epistemological stance that the human experience is "a continuous stream of consciousness" (James, 1907) that can be captured in its dynamic form within the digital realm of online experiences. Moreover, the method adopts the ontology of the moving image (Pink, 2007; Rokka \& Hietanen, 2018). Table 1, below, presents a comprehensive comparison of netnography, videography and screencast videography, highlighting the focus of each method, the opportunities they present, and their shortcomings.

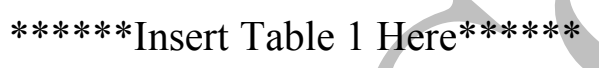

As such, screencast videography (SCV) is situated in the broader methodological realm as an extension of exploratory and observational research designs (Corbin \& Strauss, 2014) and videographic forms of inquiry (Christianson, 2016; Toraldo et al., 2016; Lemke, 2007; Belk et al., 2017; Rokka et al., 2017). It could potentially follow a style of videographic netnography that focuses on the dynamism of digital experiences, live interactions and customer journeys in the form of recorded videographic observations within the digital realm. Therefore, SCV is embedded in traditional phenomenological research and ethnographic practices that combine videographic techniques to produce detailed observational records of lived experience in its dynamic form in a digital environment. SCV is thus both an adaptation of current videographic research to suit a digital context, and an extension of netnographic research which is able to account for the dynamic live interactions and experiences and the elusive knowledge that might be obtained through screencast-based research.

The next section explains the protocol of the method and discusses the various data collection and analysis stages, as well as outlining the associated ethical considerations. 


\section{Screencast videography as a research method}

Although based on the idea of using screencasting as a means of data collection, the SCV method is not concerned simply with using screencasts. Rather, it is, as previously mentioned, a visual and dynamic method that offers detailed observational records of live on-screen experiences, capturing dynamic interactions and mapping all these interactions within experiences in the digital world. As such, it is concerned with dynamic understandings rather than static representations of experience in the digital world. Despite its focus on dynamic interactions in a digital environment, various types of SCV are possible. These relate to the contextual use of the method, as well as to types of data collection, as explained below.

\subsection{Data collection protocol}

As in videographic research, in SCV the researcher must make various decisions before and during the process of data collection, in accordance with the aims and objectives of the research. This is a visual dynamic method; therefore, the research questions must be in line with this type of research design. Based on the research questions, a series of steps must be taken before beginning the data collection:

(1) Context. Define the substantive context of the study. What is the research topic? What industry does it apply to (if applicable)?

(2) Intervention level. The most naturalistic approach will impose no limits on the individual, and therefore will not force a task on participants, but will rather allow them to create their own experiences in any way, shape or form. A less naturalistic approach may give the participants a specific task to perform during a timed screencasting session, with little room for activities beyond the specific task. Regardless of where on the spectrum of naturalistic observations the method is deployed, its dynamic affordances will still be 
invaluable in mapping the live dynamic interactions of an experience. However, such interventions should be clearly acknowledged in the research design.

(3) Software. Decide what software is to be used. Advances in technology are resulting in a wide variety of choices. The leading software package for screencasting is currently Camtasia. However, various other packages are available, including screen capture through QuickTime Play on Macbooks, and other screen capture software specifically for smartphones.

(4) Modes in screencasting. Several options are available in most screencast software.

- The choice of recording either a full screen or part of a screen. This is up to researchers to decide, based on what they are interested in capturing. As a rule of thumb, it is always best to record full screen, at least initially, in order to avoid any loss of rich data that might be outside the selected area for recording.

- The choice of audio recording using an attached or embedded microphone. Decisions should be made on whether it is important to hear the participants during screencast sessions. If screencasting occurs in the presence of the researcher, participants may comment or begin discussions during screencast sessions, so audio recording is recommended. Audio recording may also be embedded in the design of the research, for instance if participants are asked to talk to the researcher during screencasts in an auto-driving screencast videography.

- The choice of camera recording. Several screencast software packages offer the option of parallel recording, in which both the screen and the user are recorded. Using a camera that records the participant may be more obtrusive than recording the screen only, and this issue should be acknowledged if a camera is used (see point 6 below). 
(5) Timeframe. Two issues are relevant to the timeframe. The first is the length of the SCV itself. Based on the aims of the study, the researcher may decide that a time limit should be imposed, so the length of the screencast video will be predefined. A naturalistic approach will allow participants full immersion in their digital experience, without interruption or time limit; otherwise, they may begin to feel rushed to behave in a certain way. This decision should be made in light of whether the research focus requires a timeframe.

The second timeframe-related issue is the period of data collection for a project. Is the digital experience under study a seasonal one, such as online shopping during sales or on Boxing Day? If so, the data collection period will be restricted by the seasonality of the study. However, if no such limits apply, data collection may last days, months or even years, depending on the nature of the project and in line with existing recommendations on data collection in general (e.g. Corbin \& Strauss, 2014). Data collection may continue until theoretical saturation is reached, which is the point at which no additional data would result in any new insights (Creswell, 2009; Goulding, 1999).

(6) Managing obtrusiveness. The issue of obtrusiveness has been extensively debated in vidoegraphic research. Similarly, in SCV, levels of obtrusiveness may be identified and managed as necessary. Knoblauch, Tuma, and Schnettler (2006: 11) assert that the advantage of video as an observational technique is its ability to produce "natural data; data that are collected when the people studied act, behave and go about their business as they would if there were no social scientists observing or taping them". However, this perspective on natural data in videography is problematic, as Belk and Kozinets (2005: 129) suggest that "the camera can prove an unwelcome hindrance to the formation of 
interviewer-interviewee rapport. Shoving a camera in a person's face is both unnatural and obtrusive." Humans react unavoidably to a camera and, as Vom Lehn, Heath, and Hindmarsh (2002) argue, people "merely play-act when being filmed". However, this argument has been refuted by a number of scholars, who suggest that the effect of the camera may fade within a short time, after which it is possible for participants to act naturally whilst being filmed (Jayasinghe \& Ritson, 2012).

As opposed to videography, in screencasting, videos do not automatically use a camera to record the participants behind the screen; rather, as explained in the definition of screencasting, it is a "method of presenting digitally recorded playback of computer screen output which often contains audio narration" (Brown et al., 2009: 1748). Therefore, the screencast video only records screen activities, unless the researcher opts for parallel recording in which the camera will also be in action. As such, SCV need not deal with the potential influence of the camera unless the researcher makes a conscious decision to include camera recording of the participant along with the screencast. It provides less obtrusive ways of capturing digital experiences, thus allowing access to otherwise unobservable experiences. It offers the advantages of rich natural data in the form of otherwise unobservable online behaviours, but at the same time is more userfriendly and less obtrusive than camera-based videography. This is because, in a screencast, participants can see no difference on the screen while shopping, so the camera has no effect unless imposed by choice. The argument against natural data may potentially apply to screencasting, in the sense that it is unnatural for the screen itself to be recorded, or for the participant to be participating in a research project. Indeed, suggesting that SCV produces more natural data does not mean that such data are a pure 
representation of reality. As Hietanen, Rokka, and Schouten (2014) argue, all videographic work is essentially expressive, so what participants decide to do during a screencast, or when to record a screencast, influence the nature of such data.

\subsection{Analysis and interpretation}

Analysis of video data is time-consuming owing to the nature and richness of the data and the wide-ranging possibilities for analysis (Belk and Kozinets, 2005; Belk et al., 2017; Knoblauch et al., 2006; Vom Lehn et al., 2002; Christianson, 2016, Mondada, 2012). Unlike other forms of research, analysis of visual research may be tricky, and video analysis, in particular, is a messy process. Whilst the literature on visual analysis has been growing (e.g. Bell, Warren, \& Schroeder, 2014), Lemke (2007: 44) explains that yideo:

...is an even richer source of data for re-analysis than are photographs or audiotape. Not only does it include all the information that could be obtained from these two sources separately, but it provides information on the temporal relationships of speech and sounds to visually depicted actions and events.

Some of these issues may be simplified by using a form of SCV that does not include participant camera and audio narration, but when these are added to the already complex visual dynamic aspects of a screencast, it results in complex, multimodal, rich datasets that are often huge. Belk et al. (2017: 6) attribute this to "the vagaries of qualitative data analysis, which become multiplied a 1000-fold because of the potential richness of audio-visual data". This links to Rogoff's (2002) notion of the "ecology of images", in which the multimodality of images, sounds and spatial delineations are read onto and through one another, thus adding more layers of meanings.

The issue of multimodality is one of the most discussed issues in the video analysis literature. For instance, Bezemer and Jewitt (2010) discuss at length the multimodal analysis of 
video data, and the interrelationship between the visual, audial and spatial. A key distinction between Bezemer and Jewitt's (2010) approach and the possible multimodal analysis of SCV data lies in the nature of the multimodality of the screencast itself. This may include, but is not limited to, textual and visual screen content, dynamic actions on and between pages, and mouse pointer movements, as well as audio recording if this is included as part of the screencast, and camera recording where participants as well as the screencast are recorded. In the case of the latter, another multimodal video of the participant is combined with the screencast, leading to ever higher levels of multimodality. These multiple levels call for a guided approach to analysis.

Whilst traditional analysis methods, such as thematic analysis, content analysis and discourse analysis (Corbin \& Strauss, 2014), are all possible approaches to analysing video data based on the topic at hand and the research design, addressing its multimodality means such analyses must be modified for the purpose of video analysis (Belk et al., 2017).

To begin a screencast video analysis, the initial steps of video analysis apply. Daniel (2006) suggests that it should start by watching and re-watching the video repeatedly until patterns or generic themes are established. The various modes of watching videos are extremely important, for example using functions such as sound mute, slow motion, fast forward, pause and playback. Despite its simplicity, this step is vital in establishing a comprehensive view and an overall understanding of the story or stories presented in the video. Once this simplistic "feel of the data" has been established, a more sophisticated approach to analysis is required. The analytical framework to be used should be in line with the research design of the project and should account for the issues of multimodality and the dynamic nature of the method.

In studying a digital experience from James's (1907) perspective of a "continuous stream of consciousness", the dynamic nature of experience is a core quality that SCV seeks to capture. 
One analytical approach suited to this framework is critical incident analysis (Edvardsson \& Roos, 2001), which allows for efficient mapping of critical points in the experience. From a practical perspective, it is also important because, as previously mentioned, the length and multimodality of video data often makes it impossible to establish codes for analysis unless a critical incident procedure is defined. Flanagan (1954: 327) defines a critical incident as: ...any observable human activity that is sufficiently complete in itself to permit inferences and predictions to be made about the person performing the act. To be critical, an incident must occur in a situation where the purpose or intent of the act seems fairly clear to the observer and where its consequences are sufficiently definite to leave little doubt concerning its effects.

Thus, the criticality of an incident is evaluated in the context of the overall experience and its situational context. In other words, a critical incident is one in which there appear to be clear approach or avoidance consequences, or a substantial variation or turn of events in a digital experience. However, critical incident analysis results in a focus on what is critical for analysis purposes, so care must be taken to preserve the dynamic qualities of the whole experience. Critical incident analysis enables identification of critical moments in a video, making analysis of multimodal video chunks less daunting. However, very detailed analysis of every second of multimodal video data is often impossible when dealing with longer videos. A trade-off must therefore be made between detailed representation of the multimodal data, the dynamic nature of such data, and the need to gain in-depth insights into important interactions beyond mere description.

The final aspect of video analysis is the issue of representation. In visual data analysis in general, and video analysis in particular, the issue of representing the data and making the various trade-offs between maintaining richness and accomplishing simplicity is paramount. Flewitt (2006) argues that, in contrast to the process of transcription used for audio recordings, 
"Representation is a more fitting description of the interpretive processes involved in the transformation of visual, multi-method data resources into the written forms required by academic writing" (Flewitt, 2006: 34). In video analysis, representation of the data is the initial step in simplifying its complexity and presenting it in textual form. Nonetheless, as Stake and Kerr (1995: 58) comment, "all representations are, to some extent, misrepresentations"; thus, great care must be taken during video analysis to ensure that the authenticity and richness of the data are not lost. Nevertheless, some form of systematic representation is required. According to Flewitt (2006), transcription in itself is a theory, and the transformation of complex and rich data into a more simplistic form for analysis purposes not only reflects subjectively established research aims, but also inevitably directs research findings. It is important to choose the form of representation that best fits the research aims and objectives: "representations may range in style from the very complex to the quick and dirty but they need to serve the purpose for which they have been produced" (Plowman \& Stephen, 2008: 15).

The final issue of representation relates to the presentation of research findings. Although videographic research is becoming more prevalent, a simple verbal form of analysis and findings is required for most research outlets. This presents videographic researchers who use either camera videography or screencasting with the issue of maintaining the richness of data while providing verbal representations of videos without too much misrepresentation or loss of the story. To overcome this issue, a recommended technique for presenting videographic data in a text-based paper is to upload the edited and finalised critical incidents as an "unlisted video" to a video host such as YouTube or Vimeo. This option of uploading unlisted or password-protected video material means that only people who have a hyperlink to the video can view it. For instance, unlisted videos will not appear in any of YouTube's public spaces, such as channel 
pages and search results (Google, 2014). A hyperlink of the video can then be embedded in a manuscript, accompanied by a screenshot of the relevant critical incident being discussed. This method allows the reader to click on the hyperlink to watch the critical incident in its video form, accompanied by a verbal representation in text form, thus maintaining some of the richness of the video data whilst presenting the research in a journal paper.

This method should also be accompanied by a QR code for each hyperlinked video. QR codes are simple and easy to produce by copying a URL link into a QR code generator. These codes can be scanned with a smartphone in order to watch the videos directly while reading the hard copy of a videographic paper. QR codes should be placed next to a screenshot of the hyperlinked video to make it easier for the reader to view the video while reading either a soft or hard copy of the research.

\subsection{Ethical considerations}

The ethical codes and guidelines that apply to research in the social sciences in general (for details, see Corbin \& Strauss, 2014; Denzin \& Lincoln, 2011), and those of videography- and netnography-based research in particular (Belk et al., 2017; Kozinets, 2002, 2015), naturally apply to the method of SCV. Belk and Kozinets (2005) briefly discuss the ethical uses of cameras for research purposes, especially when they are installed and left for a long period, as participants may forget that they are being filmed and start to engage in behaviours that they are unaware are being recorded.

In $\mathrm{SCV}$, what is being recorded is often not the person but the screen used by the participant, unless a conscious decision is made to include a camera to record the participant as well, in which case the ethical codes of videographic research fully apply. However, the focus here is on ethical codes in SCV where participants are not present, although their voices may be recorded. 
Screencasts are recordings of screen content and any activities being performed on screen. Our screens are private spaces in the digital realm; therefore, it is important that both participant and researcher are aware of what is being recorded. Emails and social media accounts are two of the most popular types of website likely to be open on a participant's screen, so it is important to remember that SCV grants privileges that the researcher does not have. For instance, a popular debate in cyberspace research is on public versus private information, and whether forum contributions are public or private. Kozinets (2002: 65) suggests that, in establishing ethical codes for netnographic research:

...the researcher should take a cautious position on the private-versus-public medium issue. This procedure requires the researcher to contact community members and obtain their permission (informed consent) to use any specific postings that are to be directly quoted in the research.

Similarly, in SCV, informed consent must be obtained prior to data collection. While the issue of public versus private does not fully apply, in screencasts the recording is of a private screen and activities, so participants should always be aware of it, and caution should be adopted if personal data (e.g. email addresses, inboxes, private messages, etc.) appear in recordings. If such data are recorded, video editing should be performed immediately to delete all identifying and private information. Depending on the length and breadth of a project, it is possible to train long-term participants to blur and edit screencast video chunks that they do not wish to share with the researcher.

Another particularly relevant issue of SCV research is informed consent. Consent relates not only to agreeing to participate in a research project, but also, in the case of SCV, to a possible lack of anonymity. Indeed, scholars of both videography and netnography explain how such data may often reveal participants' identities (Belk \& Kozinets, 2005; Kozinets, 2015). In the case of 
SCV, the same rules apply. Participants must be aware of the potential inclusion of their captured digital experiences in research outlets, books, video links, news articles, and so on. Careful consideration must be given to the inclusion of any sensitive information, and the researcher must ensure that no harm is caused to participants as a result of their participation.

The last recommendation on the ethical use of SCV is closely linked with Kozinets's (2002) recommendation that, where possible, participants should be shown how their data appears in the final outlet, in order to ensure that they still agree to the inclusion of their digital experiences in the research. In SCV, it is a good practice to show, where possible, the edited "final" screencast video to the participants involved before publishing the findings in video form. This step aims to ensure that participants are given time to consider their participation once more, and to allow them to provide feedback prior to publication of the research.

These ethical considerations are not exhaustive, and the general ethical rules of social research involving human participants apply equally to this method. However, the considerations outlined above are specific to the proposed method and its application.

\section{Illustrative example: Screencast of purposefully purposeless experiences}

This section presents an example of how screencast videography works, and the types of insights it generates. The example explores the online fashion shopping experience and the associated customer journeys as customers interact with online fashion shopping environments. Using screencast videography enables the digital experience to be captured as it occurs, allowing a dynamic overview of the experience and detailed mapping of the many interactions involved. There is already great interest in understanding the dynamic forms of experience and customer journeys offline, not only from a research perspective but also from a managerial one (Rawson, 
Duncan and Jones, 2013; Richardson, 2010). Screencast videography enables this live dynamic capture of experience in the digital world, which has not been possible before.

In this example, following the protocol for $\mathrm{SCV}$, the following steps were taken:

(1) Context. The topic chosen for this illustrative example was online fashion shopping experiences. As previously mentioned, offline experiences in retail and shopping contexts have contributed rich insights into the field of marketing and consumer research (Kozinets et al., 2002; Borghini et al., 2009; Peñaloza, 1998). However, the same cannot be said of digital experiences, for which immersive and dynamic methodologies have yet to reach the same depth and understanding of the phenomenon. Accordingly, participants were invited to participate in the study and the procedure was explained to them. They were then instructed to embark on an online fashion shopping journey while screen recording was active.

(2) Intervention level. The aim of this case study was to capture these dynamic shopping experiences as naturally as is possible in such settings. Therefore, the participants were provided with a choice of web browser, allowing them to use the one with which they were most familiar. They were instructed to visit any fashion shopping websites freely, without imposing any limits.

(3) Software. The Camtasia software package was used to record and edit the screencast videos. The participants were shown how screencasts work, and a trial screencast recording of less than a minute was carried out prior to screencasting their online shopping experiences.

(4) Screencasting mode. The screencast was set to record the full screen, and a microphone was used to record any audio comments and "hums and hahs" by the participant. The 
rationale for activating microphone use was to allow participants to comment on what they were doing, and generally to gain a feeling for the state they were in during their experience. The user camera was disabled, as the core focus of the study was on the digitally mediated on-screen experience rather than the participants' faces.

(5) Timeframe. As the first SCV research of its kind, the participants were given freedom to decide how long their shopping experience should take. Given the exploratory nature of this first SCV, a time limit was not predefined in order to allow the experience to be captured as a whole without imposing restrictions. Setting a time limit might have hindered them from immersing themselves in the experience, or made them feel rushed to perform certain tasks or to make certain approach or avoidance decisions in their experience journeys. Therefore, the participants were left to decide for themselves how long they wished to take. There were no issues of seasonality during the data collection. Guiding principles for qualitative data collection were applied by collecting data until theoretical saturation was reached (Goulding, 1999). This resulted in 10 screencast videos ranging in length from less than 10 minutes to more than 40 minutes, with around 400 minutes of video data in total.

(6) Managing obtrusiveness. As previously mentioned, the participant camera was not activated during screencasting, since there was no need to record the participant but only to record the on-screen experience. The researcher was not involved in the screencast session, but was readily available if participants wished to converse on any aspect of their experience. Although many steps were taken to reduce obtrusiveness, the setting for the data collection was an office, rather than where the participants would naturally do their online shopping. Arguably, this may have influenced the naturalness of the data; 
however, despite this minimal level of obtrusiveness, the screencast videos show that most participants adapted to the environment and became immersed in their shopping after a couple of minutes.

The settings adopted in this example are for illustration only. As explained in Section 3.1, a variety of possible options and settings are available in SCV. Future research might use different settings in line with the research design of such projects.

\subsection{Results and discussion}

Following the data collection, the analytical framework followed a critical incident analysis approach (Edvardsson \& Roos, 2001), as well as multimodal analysis (Bezemer \& Jewitt, 2010), using the NVivo 10 package. The results of this illustrative case study capture the dynamics of the online fashion shopping experience, as well as mapping all live interactions within it. Chunks of screencast videos are embedded into this findings section.

Conceptualisations of the dynamics of digital experience were reached by uncovering the transitional states between (1) purposeful and purposeless browsing, (2) browsing and learning, and (3) learning and socialising. Moreover, SCV enabled all live interactions within the experience to be mapped by identifying all critical incidents and studying the connective and transitional states between them (see Figure 1).

***Insert Figure 1 here***

The screencast data reveal purposeful and purposeless browsing as two different experiential states. According to Lai and Yang (2000: 220), "the purpose of browsing refers to the purpose by which a browsing behavior is driven. If there is no purpose, the user may browse whatever he pleases or randomly". Browsing sessions not motivated by a given task are thus referred to as "purposeless". Purposeless browsing does not conform to purpose-driven pathways but is 
freestyle; users browse websites randomly, as they please. Such browsing behaviour has been classified as a "time wasting activity" (Lai \& Yang, 2000) that should be eliminated (Eadala \& Ratkal, 2014).

On the other hand, purposeful, task-oriented browsing is defined as an activity where shoppers navigate various routes to arrive at pre-defined destinations, browsing webpages in order to accomplish set tasks. In the context of online fashion shopping, the purpose may be as simple as: "I need a new pair of jeans", in which case the browsing is defined by seeking that item, whereas in other instances various criteria might be set, especially if the item is sought for an important occasion. For instance, "I need a new sleeveless, long maxi dress. It's got to be black, with wrapped V-neck. I prefer satin".

The screencasts capture the transitions between apparently purposeful and purposeless experiences. The video links below show the progression of one experience, and are illustrative of many similar transitions throughout the screencasts. Each video can be viewed by scanning the accompanying QR code or by clicking on the hyperlink on the accompanying image. URL links are also available as footnotes.

In Video $1^{3}$, Kat began her shopping experience purposefully, looking for a graduation ball dress:

***Insert Video 1 here***

Kat navigates the online fashion shopping environment in a purposeful fashion. She starts by explaining she is looking for a dress for her upcoming graduation ball. As she begins engaging with the filters on the website (ASOS), she goes to women - dresses and then her experience begins to prove frustrating as she progresses to various links via search filters. During her screencasts she speaks out loud explaining to me what is

\footnotetext{
${ }^{3}$ Video 1 https://www.youtube.com/watch?v=5gfFfY9F1sc
} 
going on: 'Um, OK, but I can't s... can I say? I can't really say whether or not I want it long, can I? No. Hmm, can I say I want it for ball to make it more formal? Product type! Oh dresses. Petite dress? Evening dresses aww, Oh they're all really short! [Laughing] Oh. I don't know there should be a button... Oh wait, I could actually say the colour, maybe that helps? I'm gonna be very boring and say 'black'. Party Dress? Casual Dress? Evening Dress? Am I already in 'Evening Dress'?’.

(Notes on Screencast 10)

Next, we see Kat's experience transitioning to a state of purposeless browsing following a frustrating purposeful browsing experience (see Video $2^{4}$ ).

$$
\text { *** Insert Video } 2 \text { here*** }
$$

After spending more than 10 minutes on three different websites - ASOS, Debenhams and House of Fraser - Kat's experience is almost at a standstill. She explains as she leaves the House of Fraser department store website that she would like to browse H\&M's latest collection. She comments while transitioning to this new experience: "Now, right now I'm just, like I went into different websites, and I'm just fed up, I would just go, I would just, OK, now I would just go to H\&M, latest collection, so I wouldn't even look for a dress anymore, because I'm just, I don't know, it's been frustrating so I'm looking for something, I might think I like... so I'm looking for the new arrivals, I don't know why, it just came in my head, I would just go through H\&M and see what kind of summer clothes they have”.

(Notes on Screencast 10).

Kat's experience takes an interesting path of purposeful purposelessness as she transitions from task-oriented shopping to browsing H\&M's summer collection in Video $3^{5}$ below.

$$
\text { *** Insert Video } 3 \text { here*** }
$$

\footnotetext{
${ }^{4}$ Video 2 https://www.youtube.com/watch?v=G09vIKxmOKU

${ }^{5}$ Video 3 https://www.youtube.com/watch?v=khCqjFTGxHo
} 
Kat navigates H\&M website; she looks through the new collection of summer clothes, but seems to specifically look at the 'matching outfits' section. She clicks on a few links before she comments: "I would just look and sometimes they have... um... a little and if I just... where they combine products to sell, which is very nice for me because I'm hopeless at dressing myself. I, mmm... I don't know where it is now though erm, yeah matching styles, there we go, then, I just, look like something like that, so I know, oh great, I can just get those jeans and that, I actually just buy this whole outfit, I'm sad like that". (Kat, Screencast 10)

The three videos above show transitional moments captured using screencast videography. For instance, the first video shows a purposeful experience centred around finding a suitable dress for an occasion. However, purposeful browsing is a challenging experience in the online fashion shopping context due the risks associated with buying fashion garments online, and the need to touch clothes and try them on (Perry, Blazquez, \& Padilla, 2013); ultimately, Kat's experience transitions into a form of purposeless browsing. If we accept the current literature's explanation of purposeless browsing as a form of time-wasting activity (Lai \& Yang, 2000; Eadala \& Ratkal, 2014), no further insights can be generated at this point. However, by examining what the screencast in video 3 shows, it becomes apparent that this purposeful purposelessness in Kat's experience extends to a new, previously unexplained dimension. That is, at this stage, Kat's experience becomes a kind of 'fashion education' as she uses the matching outfit algorithm to suggest a complete outfit. In this sense, the experience is defined beyond what it appears to be, as she explains that she is likely to choose the easy option of buying a full outfit in a purposeless browsing experience. 
These examples clearly show transitive states between shopping and learning about fashion, thus uncovering insights into educational types of online fashion shopping experiences in which the shopper's purposefully purposeless experiences are opportunities to learn how to build/match outfits. The learning and educational experiences revealed in purposeless browsing sessions are also illustrated in Video $4^{6}$ below:

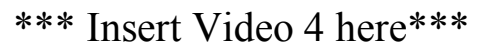

Hannah navigates the ASOS website by purposelessly looking through various sections. She then navigates to an outfits section of the website, which she shows me. It is unclear what it does, so I ask what it is; she explains as she click one product and navigates to another page showing the product details: "It's like a bit that shows you all the outfits and stuff that people have made and stuff like that, like shows you kinda looks that give you ideas. I like that, "cos then you can sort of be like "Oh I like that bag, I wouldn't know what to wear it with", that kinda thing".

(Notes on Screencast 5).

In a similar way to Kat, Hannah's experience of purposeful purposelessness features transitive moments from 'aimless browsing' to 'an opportunity for learning' regarding how to match items, or learning about new trends and ideas. This also highlights the transitive states between browsing and shopping, and between learning and socialising as learning and socialising seem to blur together, where learning comes from ideas and hacks provided by the social group (community) on the website.

\footnotetext{
${ }^{6}$ Video 4 https://www.youtube.com/watch?v=t7fTgY 8VJo
} 
Similarly, Jack's purposefully purposeless browsing of luxury fashion houses was linked to an educational need to learn about such trends and find ways to manage affordable luxury connected with self-presentation and social class (Video $5^{7}$ ).

\section{*** Insert Video 5 here ${ }^{* * *}$}

Jack's transition was from an experience of looking for shirts at Urban Outfitters to a journey of learning and inspiration as he navigated Chanel's website before moving on to Lanvin's, viewing world-class fashion shows there. He explains: "I kinda follow the main fashion houses down there, like you know kinda Prada, Chanel, what they're doing and trying [to kind of] cheap it down! [Interviewer agreeing "Aha, yeah"] you know... Like so, although I'd always like trying... umm [... participant wondering: have I spelled this wrong? Channel!] Umm, like everyone would, like kinda... I don't think everyone will do that, but I always kinda like to check what's happening on [Interviewer suggests: "Get inspired"] yeah, yeah! Get the inspiration, before... [Silence]. Umm, I find actually I'm looking at Lanvin quite a lot. Umm, you know I kinda adore their products, if I was... If I was a millionaire, I would buy it [laughing(-)]. Like again, you can see what kinda goes by it, kinda glamorous theme [music playing on the website]".

(Notes on Screencast 7)

These insights into the digital experience and transitional states between purposeful and purposeless experiences, browsing, shopping and learning represent novel contributions to the current understanding of the online fashion shopping experience. This extends the similar concept of vicarious consumption (Hartmann, Wiertz \& Arnould, 2015), but goes beyond the concept of lurking and associated consumptive moments to include, in the specific case of fashion shopping, the dimensions of sociality and learning as parts of the online shopping experience.

\footnotetext{
${ }^{7}$ Video 5 https://www.youtube.com/watch?v=7MQZYwE-LXM\&feature=youtu.be
} 
For the purposes of illustrating the use of SCV, these serve as an example of one of its many possible uses. Indeed, prior research on experience, particularly in offline contexts, has contributed in-depth insights to the understanding of various types of experiences, such as the cultural and social values connected with extraordinary experiences (Arnould et al., 1998; Scott et al., 2017; Tumbat \& Belk, 2011). Understanding of retail and shopping experiences in offline contexts has been enriched by previous work (e.g. by Kozinets et al., 2002; Borghini et al., 2009; Peñaloza, 1998). The latter was made possible by visual ethnographic work; however, a still limited understanding of online and digital experiences, particularly in retail contexts, remains apparent (Hassenzahl, 2010; Petermans, Janssens, \& Van Cleempoel, 2013). This simple case

therefore exemplifies the contributions which could be made to digital experience research by focusing on its dynamic form, emphasising its transitional moments, and mapping its critical incidents. The illustrative example shows one possible use of SCV, and future research will no doubt take this method further to generate fruitful theoretical insights into the digital realm in relation to our digital selves (Belk, 2013; Riley et al., 2015) and digital experiences.

\section{Discussion and conclusion}

In focusing on capturing computer-mediated, digitally-occurring experiences in their dynamic form, screencast videography brings valuable insights to our understanding of human experiences in the digital realm. The insights potentially enabled by this method can revolutionise our understanding of, and outlook on, online experiences. They are transformations of the capabilities currently afforded by existing methodologies, as screencast videography brings two invaluable methods - videography and netnography - into one evolved methodological approach. As was identified in the literature review, the existing research has highlighted some shortcomings of videography and netnography. Screencast videography 
addresses these, and supports the generation of previously impossible insights. These are discussed in detail below:

1. From digital footprints to full experiences

Netnography's reliance on content shared online - only digital footprints in the form of posts, comments, likes, follows, shares, etc. - is a methodological limitation manifested in various ways. First, as Lugosi and Quinton (2018) recently suggested, the mapping of interactions between human and nonhuman actors remains challenging. Screencast videography, as shown in this paper, enables interactions and experiences to be captured in their dynamic form; thus, the interactions of actors can be mapped (see Figure 1, mapping the online fashion shopping experience). Screencast videography can now create novel insights related not only to what is shared by customers, but also to their navigations, interactions and behaviours, that may not produce a datapoint capturable by a conventional netnographic approach. This paper has only given a simple illustrative example, but future screencast videography-based research is needed to unpack these digital experiences and interactions in various contexts and situations.

\section{From static to dynamic experiences}

Building on the previous point, Netnography's main mode of data collection is capturing what individuals share about their experiences, rather than the experiences themselves (e.g. García-Álvarez et al., 2017). Naturally, as Kedzior (2014) remarked, the observational methods that tend to be the crux of ethnographic work have not been applicable using netnography. Digital experiences tend to happen in our private spaces - in the comfort of our homes - or on the go, as we navigate the 'real-world', heads buried in phones. Generating insights from observing digital experiences has been impractical, and often impossible. Screencast videography offers a simple approach not only to observing but also to capturing those 
experiences in video form for further analyses and scrutiny; all via simple screencasting software or mobile apps.

While ethnography-based experience research allows fully immersive understanding and depth of meaning (see, for example, Kozinets et al., 2002; Borghini et al., 2009; Peñaloza, 1998), the same cannot be said for the limited digital experience research, which to date has mostly been design-focused and static in nature (Kawaf \& Tagg, 2017). A dynamic understanding of Hassenzahl's (2010) conception of experience as a chunk of time (during which various actors are knitted together) is necessary. The Jamesian philosophy describes experience as 'a stream of consciousness' (James, 1905), but current methodological approaches fail to capture this dynamic, instead studying digital experiences from a static perspective (Rylander, 2012). Screencast videography, embedded in the ontology of the moving image (Rokka \& Hietanen, 2018) enables recorded observations of numerous digital experiences, from shopping to dating, gambling, videogaming, and information and ideas searches. Screencast videography not only offers a dynamic form of netnography, but also extends the advantages of videography and embeds them in studying the digital realm.

3. From text to video

Lugosi and Quniton (2018) recently asserted that most netnography research focuses on textrelated interactions and conversations rather than visual ones, with a few exceptions (e.g. Arvidsson \& Caliandro, 2016; Kozinets et al., 2016). Indeed, digital environments are visual environments; simulated and animated, highly interactive, and complex (Kedzior, 2014). The power of video (Christianson, 2016; Toraldo et al., 2016; Lemke, 2007; Belk et al., 2017; Rokka et al., 2017; Rokka \& Hietanen, 2018) has rarely been employed in netnography in its current format. Screencast videography, as shown in this paper, fully adopts the power of video by using 
screencasts - the type of video most suited to reflecting the digital environment. By overcoming the limited use of video in netnography, this method can potentially generate insights into the digital experience that are "resonant, emotional, vibrant and humanizing" (Kozinets \& Belk, 2007).

4. Screencast videography as a 'vehicle of theorising'

The ways in which the insights created by screencast videography can contribute to theorisation can be explained in a variety of ways. Rokka and Hietanen (2018: 11) gave the following explanation:

How to think of videography as a vehicle of theorizing then, more concretely speaking? While descriptive theorizing common to representational mode of videography may be warranted at times, emergent theorizing seeks to embrace the immanent qualities of video, and in this sense every videography can be seen as already and necessarily a form of action research (Ozanne et al., 2017) [...] what we mean by theorizing on videography is to be both ontologically and epistemologically understood as something else we are accustomed to in traditional academic representation.

Theorising in videography research is a much-debated topic. From representational videography (Knoblauch, Schnettler, Raab \& Soeffner, 2006; Flewitt, 2006) to expressive videography (Hietanen, Rokka \& Schouten, 2014; Rokka \& Hietanen, 2018), Rokka and Hietanen (2018: 10) recently argued that theorisation in video-based research is "shorthanded by its insistence and drive towards building logical, static and mirror like representations of articulations, categorizations and concepts in textual form".

Representational modes of screencast videographies may seek to capture experiences as they occur in order to shed light on otherwise unobservable experiences, producing close representations of reality. On the other hand, screencast videography may also follow expressive 
modes of videography research. Expressive non-representational videography, as Belk et al. (2017: 436) explained, "emphasises a focus on practices, performances and daily life. Like Heitanen et al. (2014), it aims to provoke resonances, expressive passions and 'generalised affectivity"'. Expressive modes of screencast videography stem from an alternative ontological and epistemological stance similar to that of expressive videography, which aims to embrace the evocative power of video, pushing the boundaries of traditional academic research into new forms of theorising.

Belk et al. (2017: 436) commented that:

For videography to be taken as a serious scholarly endeavour in marketing, it needs to be more than simply the presentation of edited audiovisual data. If it is to extend understanding by building upon and facilitating the theoretical developments presented in other formats - such as in textual published articles - it needs to transcend the mere presentation of a story or even a complex narrative.

Screencast videography can be thought of as a vehicle of theorisation that builds on the dynamic qualities of digital experiences to truly reflect the Jamesian 'stream of consciousness' perspective of experience. As this discussion has shown, screencast videography avoids many of the limitations of the existing methodological approaches to studying digital spaces and experiences. This approach is not simply a presentation of edited audiovisual data, nor is it merely a way of representing in video form what is already established theoretically. Instead, screencast videography embeds itself in the ontology of the moving image with the specific purpose of capturing experiences in the digital realm as streams of consciousness.

To sum up, screencast videography opens up a window of opportunity for future research to enrich our understanding of digital experiences including shopping, dating, gambling, bidding, and searching for information and ideas. In addition, it can investigate complex research 
questions in contexts where consumers may be unable to put their experiences into words. In addition to the aforementioned types of digital experiences, screencast videography can address, for example, questions like: (1) how do vulnerable individuals use the digital realm to cope with or escape from their reality? This could cover mental health patients, people suffering from long term illnesses or disability, refugees, etc.; (2) how do we consumer major world crises online and on social media as they are reported on our newsfeeds?; (3) questions relating to identity and the extended self; and (4) questions relating to the fluid migration back and forth between cultures and digital and social media cultures (and the national/host country culture in the case of immigration). This is not an exhaustive list, but an indicative selection. Our ability, enabled by screencast videography, to capture dynamic experiences as they occur in the digital space should generate great insights and understandings of aspects of our digital extended selves which we might otherwise be unaware of.

In the digital world, despite rapid technological advancements, we are still at the beginning of the beginning. A hundred years from now, we will probably be to the digital realm what cavemen and cavewomen are to us today: the early inhabitants of a new world. Yet today, our lives, our passions and our experiences are quickly transforming and adapting to the digital world. Indeed, finding novel methods of studying our digital experiences is not only an opportunity, but also a duty. While it is difficult to tell what areas future research might explore using the screencast videography method, as this discussion shows, its dynamic and visual affordances make it a perfect method for studying digital experiences in various forms. This approach opens opportunities for research into the digital realm and for building a better understanding of the digital experience. 


\section{References}

Arnould, E. J., Price, L. L., \& Tierney, P. (1998). Communicative staging of the wilderness servicescape. The Service Industries Journal, 18(3), 90-115. doi:10.1080/02642069800000034

Arnould, E. J., \& Thompson, C. J. (2005). Consumer culture theory (CCT): Twenty years of research. Journal of consumer research, 31(4), 868-882.

Arvidsson, A., \& Caliandro, A. (2016). Brand public. Journal of Consumer Research, 42(5), 727-748. doi:10.1093/jcr/ucv053

Bartl, M., Kannan, V. K., \& Stockinger, H. (2016). A review and analysis of literature on netnography research. International Journal of Technology Marketing, 11(2), 165-196.

Belk, R. W. (2013). Extended self in a digital world. Journal of Consumer Research, 40(3), 477500. doi:10.1086/671052

Belk, R. W., Caldwell, M., Devinney, T. M., Eckhardt, G. M., Henry, P., Kozinets, R., \& Plakoyiannaki, E. (2017). Envisioning consumers: How videography can contribute to marketing knowledge. Journal of Marketing Management [online]. doi:10.1080/0267257X.2017.1377754

Belk, R., \& Kozinets, R. (2005). Introduction to the resonant representations issue of Consumption, Markets and Culture. Consumption Markets \& Culture, 8(3), 195-203. doi:10.1080/10253860500160395

Belk, R., \& Kozinets, R. (2012). The last picture show. Advances in Consumer Research, 38, 718-720. Available at: http://www.acrwebsite.org/volumes/v38/finalpapers/the_last_picture_show.doc (accessed 23 June 2018). 
Bell, E., Warren, S., \& Schroeder, J. E., eds (2014). The Routledge companion to visual organization. London: Routledge.

Bettman, J. R., \& Park, C. W. (1980). Effects of prior knowledge and experience and phase of the choice process on consumer decision processes: A protocol analysis. Journal of consumer research, 7(3), 234-248.

Bezemer, J., \& Jewitt, C. (2010). Multimodal analysis: Key issues. In L. Litosseliti (ed.), Research methods in linguistics (pp. 180-197). London: Continuum.

Bilgihan, A. (2016). Gen Y customer loyalty in online shopping: An integrated model of trust, user experience and branding. Computers in Human Behavior, 61, 103-113. doi:10.1016/j.chb.2016.03.014

Borghini, S., Diamond, N., Kozinets, R., McGrath, M. A., Muñiz, A., \& Sherry, J. (2009). Why are themed brandstores so powerful? Retail brand ideology at American Girl Place. Journal of Retailing, 85, 363-375. doi:10.1016/j.jretai.2009.05.003

Brown, A., Luterbach, K., \& Sugar, W. (2009). The current state of screencast technology and what is known about its instructional effectiveness. In Proceedings of the Society for Information Technology \& Teacher Education International Conference (pp. 1748-1753). Waynesville, NC: Association for the Advancement of Computing in Education.

Carù, A., \& Cova, B. (2006). How to facilitate immersion in a consumption experience: Appropriation operations and service elements. Journal of Consumer Behavior, 5(1), 4-16. doi: $10.1002 / \mathrm{cb} .30$

Chaney, D., Lunardo, R., \& Mencarelli, R. (2018). Consumption experience: past, present and future. Qualitative Market Research: An International Journal, (just-accepted), https://doi.org/10.1108/QMR-04-2018-0042 
Christianson, M. K. (2016). Mapping the terrain: The use of video-based research in top-tier organizational journals. Organizational Research Methods, 21(2), 261-287. doi:10.1177/1094428116663636

Corbin, J., \& Strauss, A. (2014). Basics of qualitative research. Chicago, IL: Sage.

Creswell, J. (2009). Research design: Qualitative, quantitative, and mixed methods approaches. Los Angeles, CA: SAGE Publications Inc.

Daniel, R. (2006). Exploring music instrument teaching and learning environments: Video analysis as a means of elucidating process and learning outcomes. Music Education Research, 8(2), 191-215. doi:10.1080/14613800600779519

Denzin, N. K., \& Lincoln, Y.S. (2011). The SAGE handbook of qualitative research. London: SAGE.

Edvardsson, B., \& Roos, I. (2001). Critical incident techniques: Towards a framework for analysing the criticality of critical incidents. International Journal of Service Industry Management, 12(3), 251-268. doi:10.1108/EUM0000000005520

Eadala, P., \& Ratkal, B. (2014). Effective user navigation through website structure improvement. International Journal of Advanced Technology and Innovative Research, 6(8), 796-799.

Flanagan, J. C. (1954). The critical incident technique. Psychological Bulletin, 51(4), 327-358. doi:10.1037/h0061470

Flewitt, R. (2006). Using video to investigate preschool classroom interaction: Education research assumptions and methodological practices. Visual Communication, 5(1), 25-50. doi:10.1177/1470357206060917 
García-Álvarez, E., López-Sintas, J., \& Samper-Martínez, A. (2017). The Social Network Gamer's Experience of Play: A Netnography of Restaurant City on Facebook. Games and Culture, 12(7-8), 650-670.

Google (2014). Video privacy settings. YouTube Help. Available at: https://support.google.com/youtube/answer/157177?hl=en (accessed 21 May 2014).

Goulding, C. (1999). Consumer research, interpretive paradigms and methodological ambiguities. European Journal of Marketing, 33(9/10), 859-873. doi:10.1108/03090569910285805

Hamilton, K. and Hewer, P. (2009), "Salsa magic: an exploratory netnographic analysis of the salsa experience", Advances in Consumer Research, 36, pp. 502-508.

Hartmann, B. J., Wiertz, C., \& Arnould, E. J. (2015). Exploring consumptive moments of valuecreating practice in online community. Psychology \& Marketing, 32(3), 319-340. doi:10.1002/mar.20782

Hassenzahl, M. (2010). Experience design: Technology for all the right reasons. Synthesis Lectures on Human-Centered Informatics, 3(1), 1-95. doi:10.2200/S00261ED1V01Y201003HCI008

Hietanen, J., Rokka, J., \& Schouten, J. W. (2014). Commentary on Schembri and Boyle (2013): From representation towards expression in videographic consumer research. Journal of Business Research, 67, 2019-2022. doi:10.1016/j.jbusres.2013.10.009

Heinonen, K. \& Medberg, G. (2018). Netnography as a tool for understanding customers: implications for service research and practice, Journal of Services Marketing, https://doi.org/10.1108/JSM-08-2017-0294 
Holbrook, M. B. (2006). Consumption experience, customer value, and subjective personal introspection: An illustrative photographic essay. Journal of business research, 59(6), 714725.

Holbrook, M. B., \& Hirschman, E. C. (1982). The experiential aspects of consumption: Consumer fantasies, feelings, and fun. Journal of Consumer Research, 9(2), 132-140. doi:10.1086/208906

James, W. (1907 [1981]). Pragmatism: A new name for some old ways of thinking. Indianapolis, IN: Hackett Publishing.

Jayasinghe, L., \& Ritson, M. (2012). Everyday advertising context: An ethnography of advertising response in the family living room. Journal of Consumer Research, 40(1), 104121. doi:10.1086/668889

Kannan, P. K., \& Li, H. A. (2017). Digital marketing: A framework, review and research agenda. International Journal of Research in Marketing, 34(1), 22-45. doi:10.1016/j.ijresmar.2016.11.006

Kawaf, F., \& Tagg, S. (2012). Online shopping environments in fashion shopping: An SOR based review. The Marketing Review, 12(2), 161-180. doi:10.1362/146934712X13366562572476

Kawaf, F., \& Tagg, S. (2017). The construction of online shopping experience: A repertory grid approach. Computers in Human Behavior, 72, 222-232. doi:10.1016/j.chb.2017.02.055

Kedzior, R. (2014), How Digital Worlds Become Material: An Ethnographic and Netnographic Investigation in Second Life, Hanken School of Economics, Helsinki. 
Kerrigan, F. and Larsen, G. and Hanratty, S. and Korta, K. (2014) Gimme shelter' : experiencing pleasurable escape through the musicalisation of running.', Marketing theory, 14 (2). pp. 147-166.

Knoblauch, H., Schnettler, B., Raab, J., \& Soeffner, H., eds (2009). Video analysis: Methodology and methods. Bern, Switzerland: Peter Lang.

Knoblauch, H., Tuma, R., \& Schnettler, B. (2006). Videography. In H. Knoblauch, B. Schnettler, J. Raab, \& H. G. Soeffner (eds), Video analysis: Methodology and methods (pp. 69-84). Bern, Switzerland: Peter Lang.

Kozinets, R. V. (2002). The field behind the screen: Using netnography for marketing research in online communities. Journal of Marketing Research, 39(1), 61-72. doi:10.1509/jmkr.39.1.61.18935

Kozinets, R. V. (2015). Netnography: Redefined. London: SAGE.

Kozinets, R., \& Belk, R. (2007). Camcorder society: Quality videography in consumer and marketing research. In R. Belk (ed.), Handbook of qualitative research: Methods in marketing (Ch. 25). Cheltenham: Edward Elgar Publishing.

Kozinets, R., Patterson, A., \& Ashman, R. (2016). Networks of desire: How technology increases our passion to consume. Journal of Consumer Research, 43(5), 659-682. doi:10.1093/jcr/ucw061

Kozinets, R. V., Scaraboto, D., \& Parmentier, M. A. (2018). Evolving netnography: how brand auto-netnography, a netnographic sensibility, and more-than-human netnography can transform your research. Journal of Marketing Management, 34(3-4), 231-242, doi: $10.1080 / 0267257 X .2018 .1446488$ 
Kozinets, R. V., Sherry, J. F., DeBerry-Spence, B., Duhachek, A., Nuttavuthisit, K., \& Storm, D. (2002). Themed flagship brand stores in the new millennium: Theory, practice, prospects. Journal of Retailing, 78(1), 17-29. doi:10.1016/S0022-4359(01)00063-X

Lai, H., \& Yang, T.-C. (2000). A system architecture for intelligent browsing on the Web. Decision Support Systems, 28(3), 219-239. doi:10.1016/S0167-9236(99)00087-1

Lemke, J. L. (2007). Video epistemology in-and-outside the box: Traversing attentional spaces. In R. Goldman-Segall, \& R. Pea (eds), Video research in the learning sciences (pp. 3952). Mahwah, NJ: Erlbaum.

Lugosi, P., \& Quinton, S. (2018). More-than-human netnography. Journal of Marketing Management, 34(3-4), 287-313.

McLean, G., \& Wilson, A. (2016). Evolving the online customer experience... is there a role for online customer support? Computers in Human Behavior, 60, 602-610. doi:10.1016/j.chb.2016.02.084

Mendeley (2014). Getting started with Mendeley. [video] Available at: https://www.youtube.com/watch?v=Gv6_HuCYExM (accessed 23 June 2018).

Mondada, L. (2012). Video analysis and the temporality of inscriptions within social interaction: The case of architects at work. Qualitative Research, 12(3), 304-333. doi:10.1177/1468794112438149

Pantano, E., \& Priporas, C. V. (2016). The effect of mobile retailing on consumers' purchasing experiences: A dynamic perspective. Computers in Human Behavior, 61, 548-555. doi:10.1016/j.chb.2016.03.071 
Peñaloza, L. (1998). Just doing it: A visual ethnographic study of spectacular consumption behavior at Nike Town. Consumption, Markets and Culture, 2(4), 337-400. doi:10.1080/10253866.1998.9670322

Perry, P., Blazquez, M., \& Padilla, S. (2013). Translating the need for touch to online fashion shopping via digital technology. Paper presented at the First International Conference on Digital Technologies for the Textile Industries, University of Manchester, Manchester, 5-6 September.

Petermans, A., Janssens, W., \& Van Cleempoel, K. (2013). A holistic framework for conceptualizing customer experiences in retail environments. International Journal of Design, $\quad 7(2), \quad 1-18$ Available at: http://www.ijdesign.org/index.php/IJDesign/article/viewFile/1185/568 (accessed 23 June 2018).

Pine, B. J., \& Gilmore, J. H. (1998). Welcome to the experience economy. Harvard Business Review, 76, 97-105. Available at: https://hbr.org/1998/07/welcome-to-the-experienceeconomy (accessed 23 June 2018).

Pink, S. (2007). Walking with video. Visual Studies, 22(3), 240-252. doi:10.1080/14725860701657142

Pink, S. (2011). Sensory digital photography: Re-thinking 'moving'and the image. Visual Studies, 26(1), 4-13.

Plowman, L., \& Stephen, C. (2008). The big picture? Video and the representation of interaction. British Educational Research Journal, 34(4), 541-565. doi:10.1080/01411920701609422

Prensky, M. (2001). Digital natives, digital immigrants: Part 1. On the Horizon, 9(5), 1-6. doi:10.1108/10748120110424816 
Rawson, A., Duncan, E., \& Jones, C. (2013). The truth about customer experience. Harvard Business Review, 91(9), 90-98.

Richardson, A. (2010). Using Customer Journey Maps to Improve Customer Experience. Harvard Business Review, 15.

Riley, S., Evans, A., Griffin, C., Morey, Y., \& Murphy, H. (2015). Crossing into the digital realm. The Psychologist, 28(8), 652-655. Available at: https://thepsychologist.bps.org.uk/volume-28/august-2015/crossing-digital-realm (accessed 23 June 2018).

Rogoff, I. (2002). Studying visual culture. In N. Mirzoeff (ed.), The visual culture reader, 2nd edn. (pp. 24-36). London: Routledge.

Rokka, J., \& Hietanen, J. (2018). On positioning videography as a tool for theorizing. Recherche et Applications en Marketing (English Edition), 2051570718754762.

Rokka, J., Hietanen, J., \& Brownlie, D. (2017) Screening marketing: Videography and the expanding horizons of filmic research. Journal of Marketing Management (call for papers). Available at: http://www.jmmnews.com/screening-marketing/ (accessed 2 January 2017).

Rokka, J., Rousi, P., \& Hämäläinen, V. (2014). Follow me on dead media: Analog authenticities in alternative skateboarding scene. Video presented at Advances in Consumer Research Film Festival, Baltimore, NJ, 23-26 October. Available at: https://vimeo.com/105879922 (accessed 23 June 2018).

Rose, S., Clark, M., Samouel, P., \& Hair, N. (2012). Online customer experience in e-retailing: An empirical model of antecedents and outcomes. Journal of Retailing, 88(2), 308-322. doi:10.1016/j.jretai.2012.03.001 
Rose, S., Hair, N., \& Clark, M. (2011). Online customer experience: A review of the business-toconsumer online purchase context. International Journal of Management Reviews, 13(1), 24-39. doi:10.1111/j.1468-2370.2010.00280.x

Rylander, A. (2012). Pragmatism and design research: An overview. Stockholm, Sweden: Designfakultetens kunskapssammanställningar.

Schembri, S., \& Boyle, M. V. (2013). Visual ethnography: Achieving rigorous and authentic interpretations. Journal of Business Research, 66(9), 1251-1254. doi:10.1016/j.jbusres.2012.02.021

Schmitt, B. (1999). Experiential marketing. Journal of Marketing Management, 15(1-3), 53-67. doi:10.1362/026725799784870496

Scott, R., Cayla, J., \& Cova, B. (2017). Selling pain to the saturated self. Journal of Consumer Research, 44, 22-43. doi:10.1093/jcr/ucw071

Stake, R., \& Kerr, D. (1995). René Magritte, constructivism, and the researcher as interpreter. Educational Theory, 45(1), 55-61. doi:10.1111/j.1741-5446.1995.00055.x

Thompson, C. J., Locander, W. B., \& Pollio, H. R. (1989). Putting consumer experience back into consumer research: The philosophy and method of existential-phenomenology. Journal of Consumer Research, 16(2), 133-146. doi:10.1086/209203

Toraldo, M. L., Islam, G., \& Mangia, G. (2016). Modes of knowing: Video research and the problem of elusive knowledges. Organizational Research Methods, 21(2), 438-465. doi:10.1177/1094428116657394

Tumbat, G., \& Belk, R. W. (2011). Marketplace tensions in extraordinary experiences. Journal of Consumer Research, 38, 42-61. doi:10.1086/658220 
Vom Lehn, D., Heath, C., \& Hindmarsh, J. (2002). Video based field studies in museums and galleries. Visitor Studies Today, 5(3), 15-23.

We are Social (2018). Digital in 2018: Global overview. New York, NY: We Are Social. Available at: https://wearesocial.com/blog/2018/01/global-digital-report-2018 (accessed 23 June 2018).

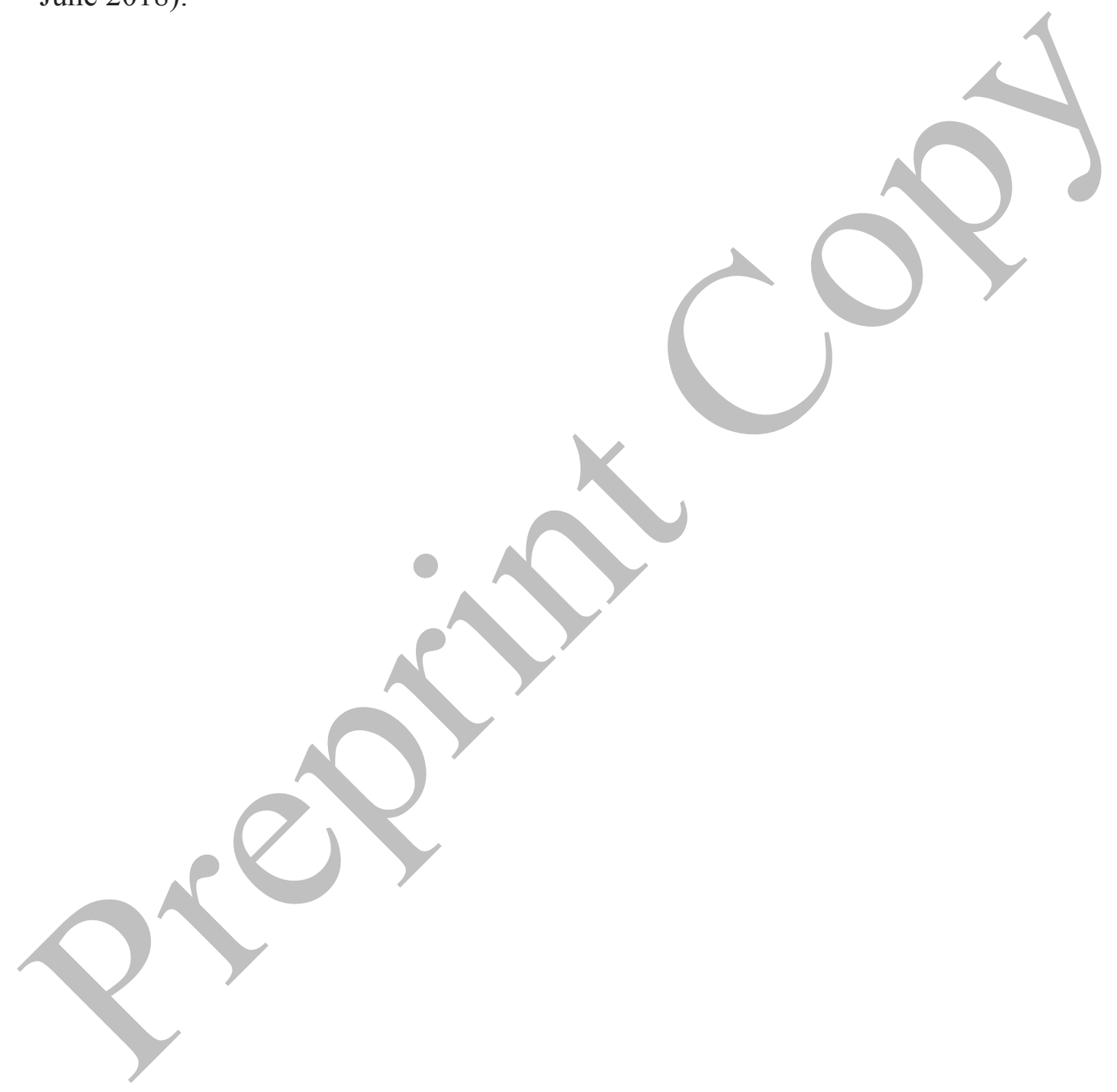




\section{Figure 1:}

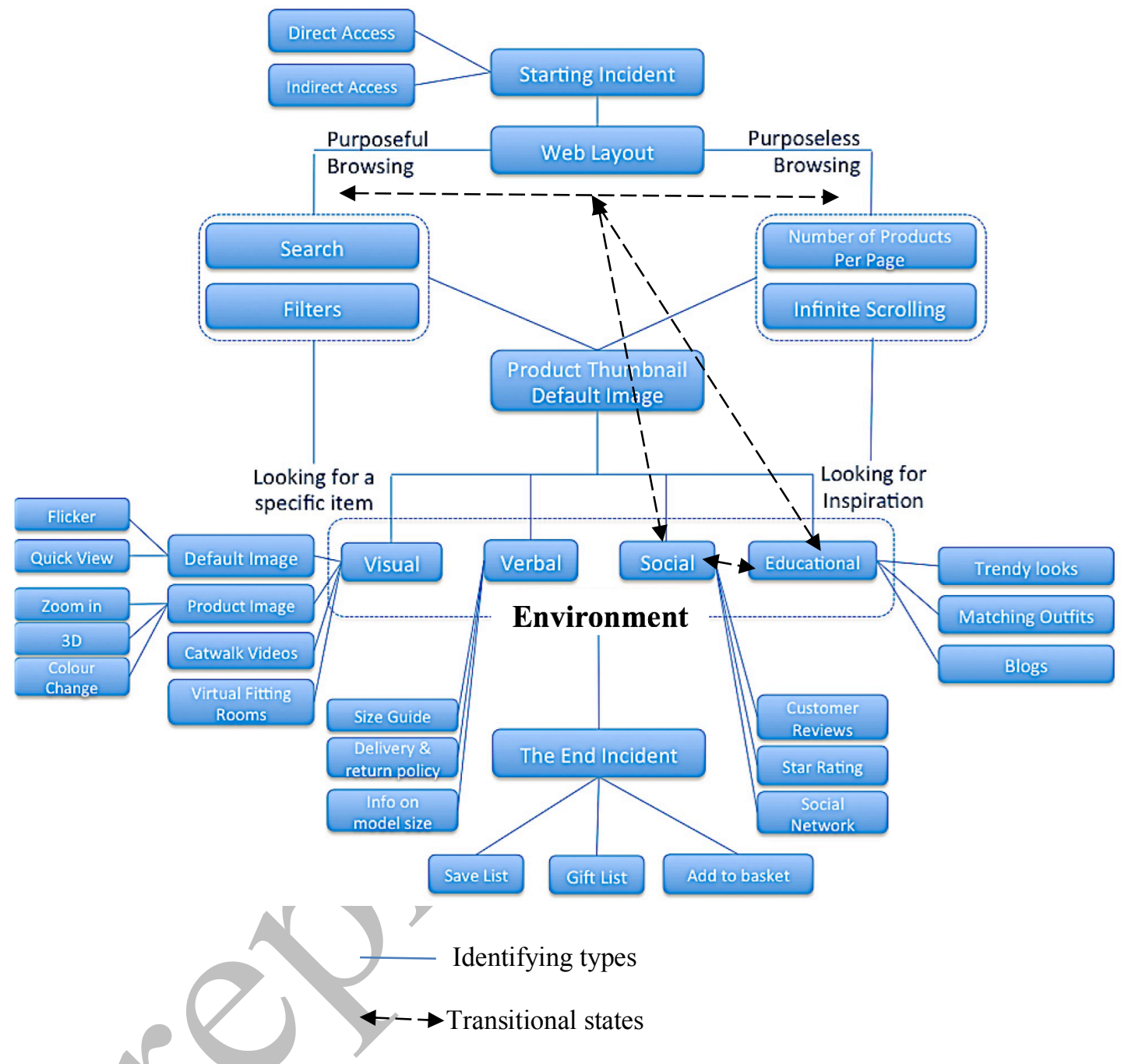

Figure 1. Mapping the online fashion shopping experience and critical incidents 
Video 1:

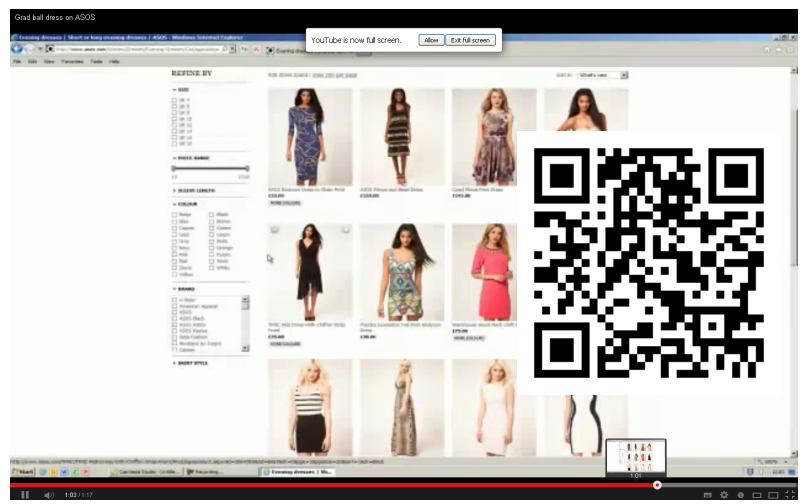

Video 1. Purposeful browsing - Grad ball dress

Video 2:

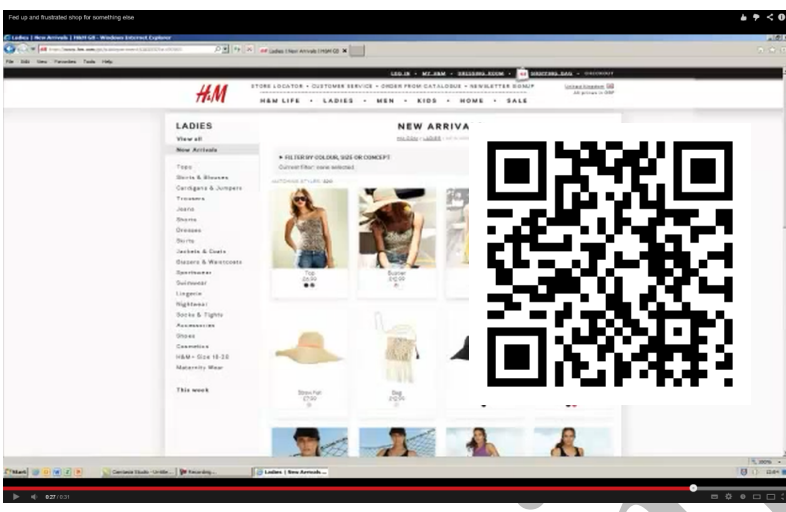

Video 2. Transitioning to purposeless browsing

Video 3:

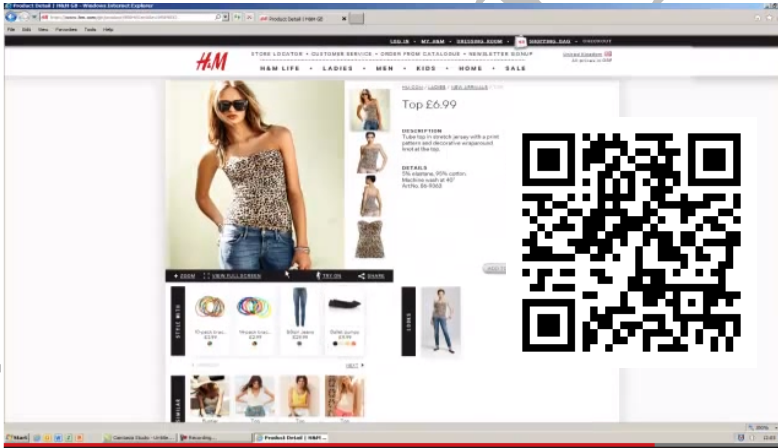

II (1) 0:41/0:50

은

Video 3. Matching outfits 
Video 4:

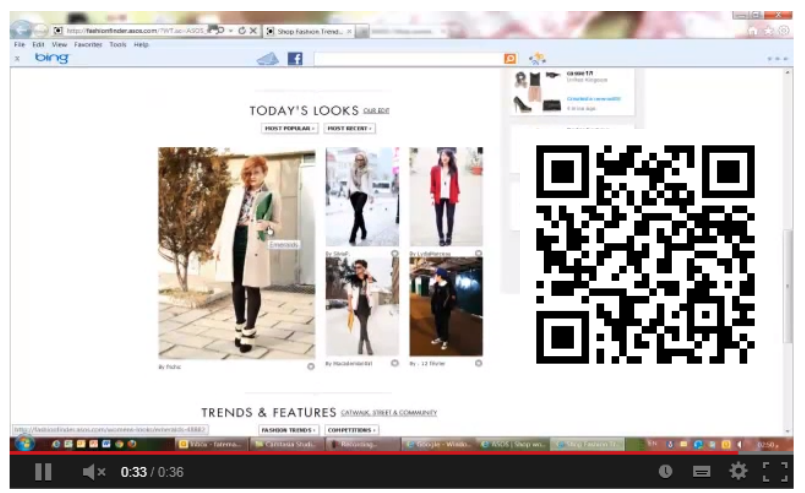

Video 4. Learning state

Video 5:

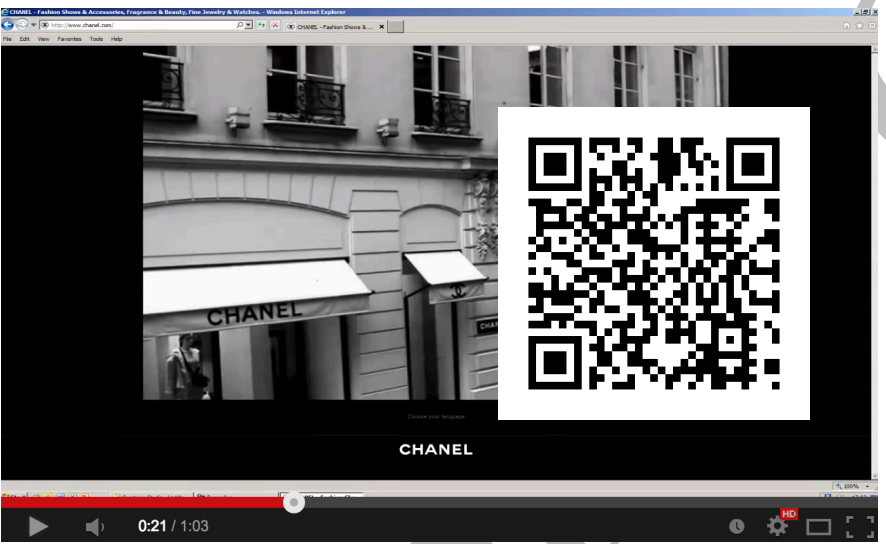

Video 5. Social and educational state 
Table 1 Comparing Videography, Netnography and Screencast Videography

\begin{tabular}{|c|c|c|c|}
\hline Attribute & Screencast Videography & Videography & \\
\hline $\begin{array}{l}\text { Definition and } \\
\text { Philosophical } \\
\text { Underpinnings }\end{array}$ & $\begin{array}{l}\text { Screencast videography is a research } \\
\text { method that adopts a dynamic visual form } \\
\text { of inquiry. It is philosophically underpinned } \\
\text { by the ontology of the moving image. The } \\
\text { method uses screencasts - videos of screen } \\
\text { activities or outputs - as its main mode of } \\
\text { data collection. The screencast videos } \\
\text { capture dynamic on-screen interactions and } \\
\text { experiences as they occur. This means that } \\
\text { detailed records of online experiences (e.g. } \\
\text { online shopping, information search, dating, } \\
\text { video gaming, gambling, among others) can } \\
\text { be offered that are not usually observable } \\
\text { using conventional methods. }\end{array}$ & $\begin{array}{l}\text { Video offers "a space of visible and audible } \\
\text { dynamic activity" (Lemke, 2007: 40). Its } \\
\text { multimodality provides a scaffold basis for } \\
\text { translating the tacit, aesthetic and embodied } \\
\text { aspects of experience into elusive } \\
\text { knowledge (Toraldo et al., 2016), offering } \\
\text { "a sense of understanding that is novel and } \\
\text { interesting, evocative and emotionally } \\
\text { engaging" (Belk et al., 2017: } 3 \text { ). } \\
\text { "The main preoccupation of videography } \\
\text { should - and must - be theoretical } \\
\text { argumentation and elaboration through } \\
\text { Hietenan, 2018: } 8 \text { ). }\end{array}$ & $\begin{array}{l}\text { Netnography is "a qualitative research } \\
\text { methodology that adapts ethnographic } \\
\text { research techniques to study the cultures } \\
\text { and communities that are emerging through } \\
\text { computer-mediated communications" } \\
\text { (Kozinets, 2002: } 62 \text { ). Its updated, redefined } \\
\text { form refers to "a new approach to } \\
\text { conducting ethical and thorough } \\
\text { ethnographic research that combines } \\
\text { archival and online communications work, } \\
\text { participation and observation, with new } \\
\text { forms of digital and network data } \\
\text { collection, analysis and research } \\
\text { representation" (Kozinets, } 2015: 1 \text { ). }\end{array}$ \\
\hline Focus & Computer mediated or digitally occurring & Dynamic and live interactions; & A window onto the digital realm. \\
\hline
\end{tabular}




\begin{tabular}{|c|c|c|c|}
\hline & $\begin{array}{l}\text { experiences; } \\
\text { Dynamic digital live interactions; } \\
\text { On screen activities and behaviours; } \\
\text { Movement and dynamics in digital } \\
\text { environments. }\end{array}$ & $\begin{array}{l}\text { Movement of persons and products in wider } \\
\text { environments; } \\
\text { Cultural and societal relations and } \\
\text { connections. }\end{array}$ & $\begin{array}{l}\text { Digital interactions and experiences. } \\
\text { Cultures and Communities in online } \\
\text { environments. }\end{array}$ \\
\hline Advantages & $\begin{array}{l}\text { Combines the advantages of both } \\
\text { Netnography and videography. } \\
\text { Allows a visually dynamic understanding of } \\
\text { communities, groups, forums as well as } \\
\text { individuals. }\end{array}$ & $\begin{array}{l}\text { Rich ethnographic videographies bring } \\
\text { resonance and compelling experiences to } \\
\text { light. } \\
\text { Visual and dynamic qualities. } \\
\text { Dynamic/moving form of knowledge is }\end{array}$ & $\begin{array}{l}\text { Provides in-depth understanding of online } \\
\text { communities, groups and forums. } \\
\text { Captures conversations online. } \\
\text { Has the ability to study social media } \\
\text { interactions. }\end{array}$ \\
\hline
\end{tabular}




\begin{tabular}{|c|c|c|c|}
\hline & $\begin{array}{l}\text { Representational and expressive forms are } \\
\text { possible. } \\
\text { Offers dynamic and moving forms of } \\
\text { knowledge, and the ability to study } \\
\text { interactions as they occur. } \\
\text { Works not only with freely-shared data, but } \\
\text { also with the behaviours of individuals } \\
\text { behind the screen that might not result in a } \\
\text { sharable trace. } \\
\text { A humanist approach in which small data } \\
\text { matter. }\end{array}$ & $\begin{array}{l}\text { possible. } \\
\text { Representational and expressive forms of } \\
\text { videography are possible. } \\
\text { A basis for translating elusive knowledge } \\
\text { and sensory ways of knowing. }\end{array}$ & $\begin{array}{l}\text { Takes a humanist approach in which small } \\
\text { data matter. } \\
\text { Works with freely shared data which is } \\
\text { often publicly available (or in limited access } \\
\text { digital spaces, e.g. closed groups, members- } \\
\text { only forums, etc.). }\end{array}$ \\
\hline
\end{tabular}




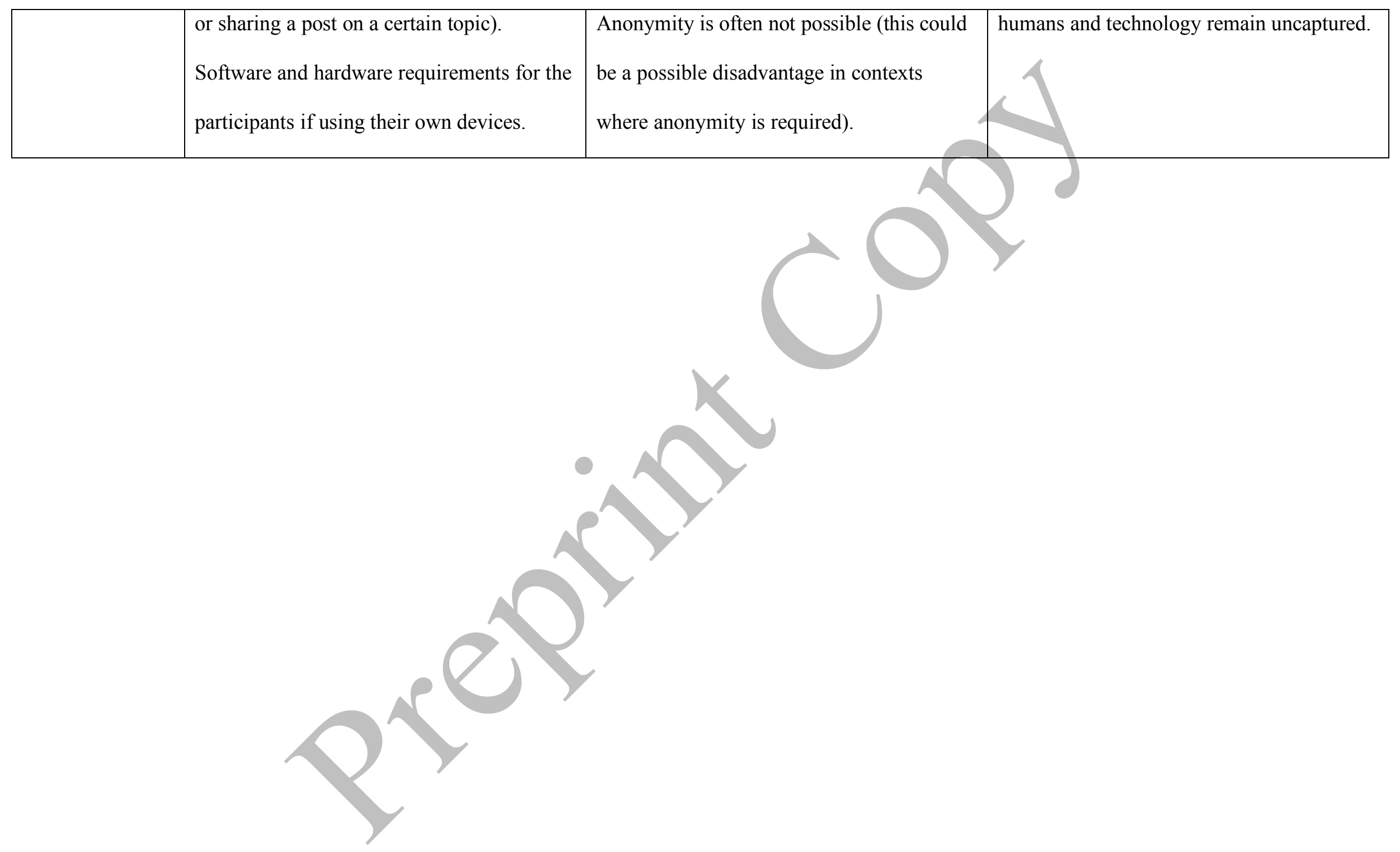

